\title{
Recovery of Laryngeal Closure in Post-stroke Survivors
}

\author{
Kaylee Sienza', Youngsun Kim² ${ }^{2}$ Taeok Park' Byung-Mo Oh \\ ${ }^{1}$ Communication Sciences and Disorders, Illinois State University, Normal, IL; ${ }^{2}$ Communication Sciences and Disorders, Ohio University, Athens, Ohio, \\ United States; ${ }^{3}$ Department of Rehabilitation Medicine, Seoul National University College of Medicine, Seoul, Korea
}

Objectives: The purpose of this study was to examine whether traditional swallowing intervention improves the initiation or duration of laryngeal closure in post-stroke survivors using the initial and follow-up videofluoroscopic swallowing study (VFSS).

Methods: Twelve post-stroke survivors completed traditional swallowing intervention such as postural changes and swallowing exercises. The initial VFSS was performed at the acute or subacute stage and the follow-up VFSS occurred at 3 to 5 weeks after the initial VFSS. Temporal measurements of laryngeal closure were analyzed for $2 \mathrm{~mL}$ and $5 \mathrm{~mL}$ thin liquids. Statistical comparisons were made by paired t-test. The significance level was set at $p<0.05$.

Results: The initiation of laryngeal closure of post-stroke survivors was significantly shorter after the traditional swallowing intervention; however, the duration of laryngeal closure did not differ after the intervention. In addition, the post-stroke survivors showed reduced occurrences of penetration or aspiration after the intervention.

Conclusions: Shorter initiation of laryngeal closure after the intervention indicates that the traditional swallowing intervention at the acute or subacute stage of stroke may help poststroke survivors protect the airway effectively.

Keywords: Laryngeal closure, Stroke, Swallowing, Recovery

\section{INTRODUCTION}

Thirty to $70 \%$ of post-stroke survivors suffer from dysphagia [1,2]. Post-stroke survivors with dysphagia commonly show difficulties in airway protection during pharyngeal swallowing $[3,4]$. Airway protection during swallowing is achieved through laryngeal closure. Laryngeal closure is executed by an initial downward displacement of the epiglottis with a concurrent approximation of the arytenoids to the base of the epiglottis [5-7]. The contact between the epiglottis and arytenoid is sustained until the bolus passes the pharynx and enters the esophagus. A delayed, incomplete, and reduced laryngeal closure may lead to aspiration. Aspiration is defined as the bolus entering into the vestibule and then passing below the vocal folds [8].

Disturbances in the initiation and duration of laryngeal closure in post-stroke survivors are important physiological steps in the pharyngeal swallow $[9,10]$. Park and her colleagues (2010) reported that the post-stroke survivors show a reduced duration of laryngeal closure regardless of aspiration [9]. However, in regard to the initiation of laryngeal closure, post-stroke survivors with aspiration showed a delayed initiation of la-

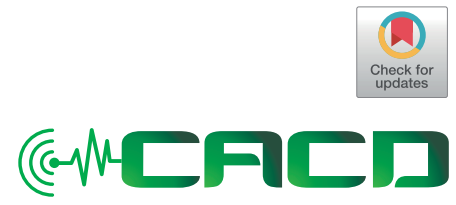

Received: April 9, 2018 Accepted: August 22, 2018

Correspondence: Taeok Park

Communication Sciences and Disorders Illinois State University, 215F Fairchild Hall, Campus Box 4720, Normal, IL 61790-4720, United States

Tel: +1-309-438-5704

Fax: +1-309-438-5221

E-mail: tpark12@ilstu.edu

(C) 2018 The Korean Association of SpeechLanguage Pathologists

This is an Open Access article distributed under the terms of the Creative Commons Attribution NonCommercial License (http://creativecommons.org/ licenses/by-nc/4.0/) which permits unrestricted noncommercial use, distribution, and reproduction in any medium, provided the original work is properly cited. 
ryngeal closure when compared to those without aspiration. Inappropriate initiation and duration of laryngeal closure are likely to place individuals at risk for aspiration during swallowing [9]. Previous studies have focused on temporal characteristics of dysphagic patients after the incidence of stroke using the initial diagnostic examination [10-12]. It is necessary to examine whether swallowing intervention improves initiation or duration of laryngeal closure in post-stroke survivors.

Several investigations have reported the progress of swallowing function for stroke survivors [13-15]. These studies examined the presence of swallowing problems following stroke at the acute stage and at a 1 to 3 months follow-up using clinical observation. They reported that the frequency of aspiration decreased, or the cough response was presented in poststroke survivors who showed silent aspiration in the initial examination. Post-stroke survivors experienced in the most progress in airway protection between 3 and 6 months; however, $40 \%$ of severe post-stroke survivors still showed aspiration after 6 months [15-17]. Therefore, it is necessary to examine whether swallowing intervention improves the initiation or duration of laryngeal closure using objective measurements of swallowing in post-stroke survivors. The purpose of this study is to refine the results of previous study using objective methods for the temporal measurements. The purpose of this study was to examine whether traditional swallowing intervention improves the initiation or duration of laryngeal closure in post-stroke survivors using the initial and follow-up videofluoroscopic swallowing study (VFSS).

\section{METHODS}

\section{Subjects}

VFSS data was collected at Seoul National University Hospital, Seoul Korea. 113 post-stroke survivors were referred for a VFSS from January 2010 to July 2014. Among these patients, 12 post-stroke survivors (mean age 63.8, 7 males and 5 females) were selected using the following inclusion criteria: (1) no history of previous stroke or other disease that could affect swallowing function, (2) initial VFSS performed during the acute or subacute stage, (3) stroke survivors had traditional swallowing intervention after initial VFSS, and (4) at least one follow-up VFSS performed 3-5 weeks after the initial VFSS. All VFSS were a part of a standardized routine diagnostic examination at Seoul National University Hospital. Stroke diagnoses were provided by neurologists and confirmed by computed tomography (CT) or magnetic resonance imaging (MRI). For the lesion, 6 patients had a unilateral middle cerebral artery (MCA) infarction, 2 patients had a unilateral corona radiata infarction, and 4 patients had unilateral basal ganglia intracerebral hemorrhage. All patients were referred from a physician to participate in the VFSS. The traditional swallowing intervention was conducted with using routine protocol at Seoul National University Hospital and was recommended based on the findings of the initial VFSS [18]. Post-stroke survivors had a 30-minute daily treatment session with a swallowing clinician that included exercises, postural changes, and diet modifications. For instances, the postural changes consisted of a chin down position and exercises included oral motor exercises and tongue strengthening exercises. Diet modification included thickened liquid or puree consistency, and a nutritionist were involved. This study of temporal measurements using videofluoroscopic films was approved by Institutional Review Board (IRB) at Illinois State University, United States and Seoul National University Hospital, South Korea.

\section{VFSS}

VFSS was conducted on all post-stroke survivors. Each poststroke survivor was seated upright in a wheelchair or stretcher chair for the examination. The fluoroscopic tube was focused in the lateral plane on the oral cavity (the lips anteriorly to the pharyngeal wall posteriorly) and the nasopharynx (superiorly) to below the upper esophageal sphincter area (inferiorly). Boluses were presented in $2 \mathrm{~mL}$ and $5 \mathrm{~mL}$ thin liquid. The thin liquid was a mixture of water and barium sulfate powder $(35 \% \mathrm{w} / \mathrm{v})$. Each post-stroke survivor swallowed the bolus after putting the liquid in his/her mouth after the clinician delivered it by spoon. A total of 48 swallows were submitted for analysis for this investigation. Frame-by-frame images were acquired through digital imaging files using a computerbased image processing system equipped with a digital computer frame grabber board (Pegasus HD/SD Board, Grass Valley Inc., Honorine, France) and image processing software (EDIUS 4.5, Grass Valley Inc., Montreal, Quebec, Canada). The X-ray voltage was set at a $40-\mathrm{kV}$ peak, which allowed the soft tissue of the pharynx to be visualized. The initial VFSS was performed during the acute or subacute stage of stroke and the follow-up VFSS was performed 3 to 5 weeks (average 28.4 days) after initial VFSS.

\section{Procedure for temporal measurement}

To accurately analyze the temporal sequence of events, slow 

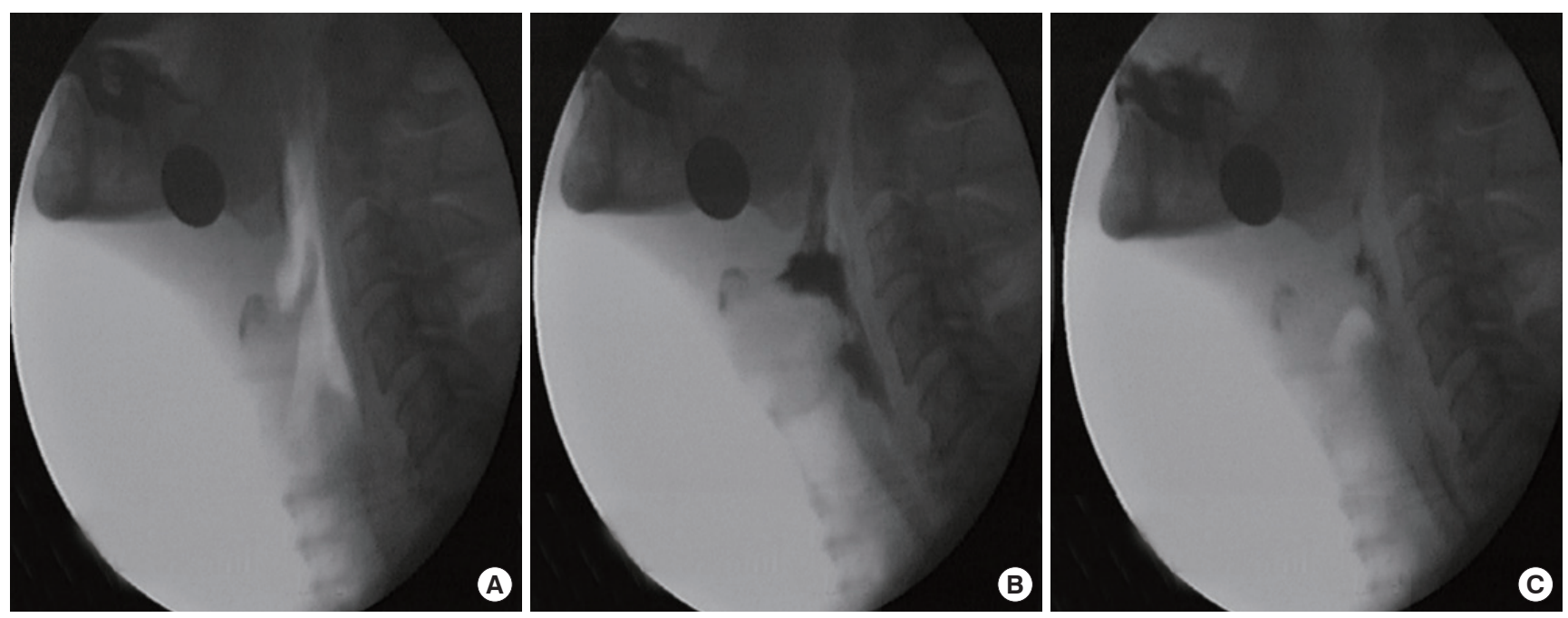

Figure 1. Tracing of a lateral view from videofluoroscopic frame of a swallow study with marked point of temporal measurements. (A) Bolus passes the ramus of mandible, (B) first contact of arytenoids and epiglottis, (C) final contact of arytenoids and epiglottis; Initiation of Laryngeal Closure (ILC): B-A. Laryngeal Closure Duration (LCD): C-B.

motion and frame-by-frame analyses were performed using a 100 -ms video timer. Adobe Element 14 (San Jose, CA) was used. First, each liquid swallow was analyzed for the following points of occurrence: (1) bolus passing the ramus of mandible, (2) first contact of arytenoids and epiglottis, and (3) final contact of arytenoids and epiglottis. Second, the times for each of the above-mentioned markers were recorded and used to calculate the two measures of laryngeal closure (Figure 1). Laryngeal closure in this investigation referred to laryngeal vestibule closure rather than true vocal folds closure. However, laryngeal vestibule closure has been and continues to be the accepted and encouraged methodology for analyzing temporal measure of laryngeal closure [19-22]. The occurrence of penetration and aspiration were verified. Aspiration was defined as entry of the bolus below the true vocal folds. As aspiration was identified, the occurrence of cough response was recorded. Penetration was defined as entry of the bolus in the vestibule, but not below the true vocal folds.

\section{Statistical Analysis}

Statistical comparisons between initial and follow-up measurements of laryngeal closure were made by paired t-test. The significance level was set at $p<0.05$.

\section{RESULTS}

\section{Reliability}

For interjudge reliability, a second independent judge ana- lyzed the designated swallows of 3 randomly selected subjects (12 swallows, 25\%). The measurements of the principal investigator and second judge were compared using intraclass correlation coefficient. A significant correlation was observed, (ICC $=0.99, p<0.01$ ). For intrajudge reliability, the principal investigator reanalyzed the same 3 subjects a second time. Intrajudge reliability was also significant $(\mathrm{ICC}=0.99, p<0.01)$.

\section{Occurrence of aspiration and penetration}

Five post-stroke survivors showed silent aspiration in the initial VFSS. Among these, four post-stroke survivors did not show aspiration, and one post-stroke survivor showed silent aspiration in the follow-up VFSS. Five post-stroke survivors showed penetration in initial VFSS. Among these, three poststroke survivors did not show penetration or aspiration and two post-stroke survivors showed penetration in the followup VFSS. Two post-stroke survivors did not show any penetration or aspiration in the initial VFSS and showed penetration in the follow-up VFSS.

\section{Initiation of laryngeal closure (ILC)}

The mean of initiation of laryngeal closure in the follow-up VFSS was significantly shorter than the initial VFSS $(t(28)=$ 2.181, $p=0.038)$. It indicated that post-stroke survivors showed an earlier initiation of laryngeal closure after the traditional swallowing intervention. The mean of the initiation of laryngeal closure in the initial VFSS was 0.80 seconds and the standard deviation was 0.76 (Figure 2). In the follow-up VFSS, 


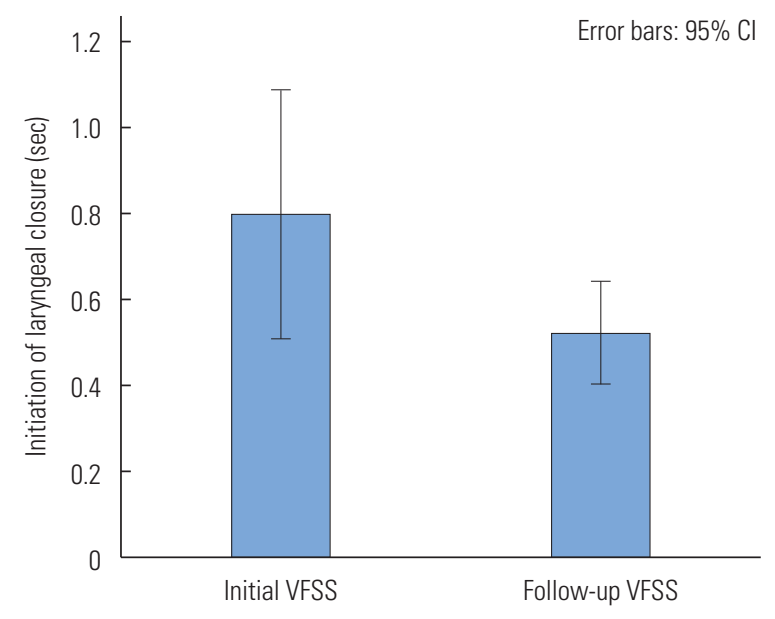

Figure 2. Means and 95\% confidence interval of ILC for initial and followup VFSS.

the mean was 0.53 seconds and the standard deviation was 0.31 (Figure 2).

\section{Laryngeal Closure Duration (LCD)}

LCD was not significantly different between the initial and follow-up VFSS $(t(32)=-1.716, p=0.096)$.

The mean of laryngeal closure duration in the initial VFSS was 0.48 seconds and the standard deviation was 0.14 . In the follow-up VFSS, the mean of the laryngeal closure duration was 0.51 seconds and the standard deviation was 0.12 (Figure 3 ).

\section{DISCUSSION}

The purpose of this study was to examine the improvement of laryngeal closure in post-stroke survivors after traditional swallowing intervention. The most significant finding was that there was a significantly shorter initiation of laryngeal closure in post-stroke survivors after the traditional swallowing intervention when compared to the initial VFSS. The findings of initiation of laryngeal closure (ILC) may indicate that traditional swallowing intervention helps post-stroke survivors better protect their airway. This improvement may be related to sensory receptor responses in the oropharynx that aid in laryngeal closure. Shorter ILC involves quickly detecting the passing bolus in the anterior facial pillar of the oral cavity which helps initial hyolaryngeal excursion, and subsequently laryngeal closure. Additionally, the initiation of laryngeal closure in this study clearly improved in the small volume of liquid. The mean ILC of $2 \mathrm{~mL}$ thin liquid in the initial VFSS was 0.92 seconds, but the mean ILC of $2 \mathrm{~mL}$ thin liquid after inter-

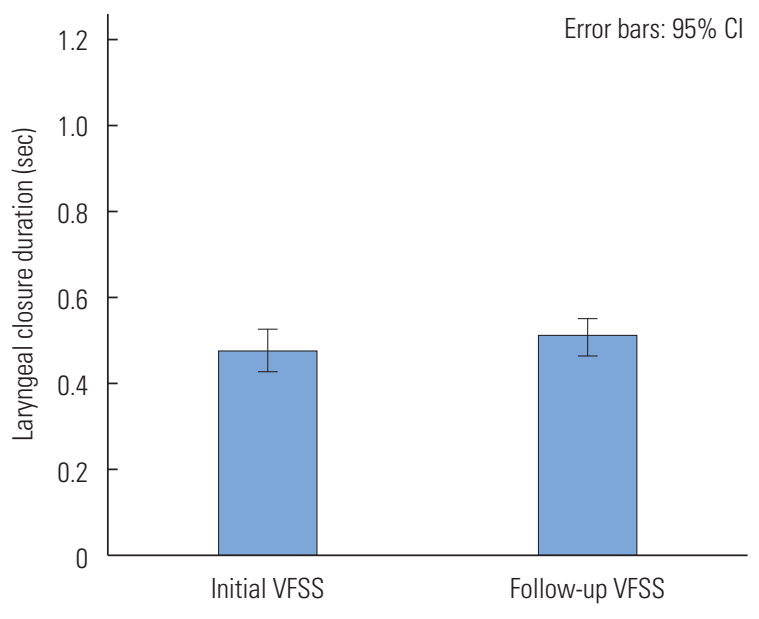

Figure 3. Means and 95\% confidence interval of LCD for initial and followup VFSS.

vention was 0.46 seconds. This may indicate that the poststroke survivors may be highly sensitive to a small volume of liquid during the swallow. The initiation of airway protection will represent the recovery in sensory receptors in the oropharynx and brain.

Short initiation of laryngeal closure in post-stroke survivors occurs earlier after swallowing intervention. Shorter ILC in post-stroke survivors may reduce a risk of penetration and aspiration before and during the swallow. If the larynx closes quickly before the bolus reaches the larynx during swallowing, the bolus is less likely to enter the vulnerable airway. Four post-stroke survivors in this study showed no aspiration and three post-stroke survivors showed either aspiration or penetration in the follow-up VFSS. Four post-stroke survivors with silent aspiration in the initial VFSS did not show aspiration after intervention. This finding is similar to the results of previous studies. Terre and Mearin (2006) reported that penetration and aspiration in post-stroke survivors reduced from the baseline and follow-up after one month of intervention [16]. The post-stroke survivors with silent aspiration were reduced from $35 \%$ to $27 \%$ when comparing the first examination with an examination that followed one month later. Additionally, Seo et al. (2011) reported that the post-stroke survivors, who showed aspiration at the initial VFSS but not at the follow-up VFSS, showed improvement of epiglottis movement involving the contact between the arytenoids and epiglottis in order to close the larynx which prevents aspiration [18].

The effects of traditional swallowing intervention in poststroke survivors showed promising results in this study. The traditional swallowing intervention of this study included diet 
modifications, postural changes, thermal stimulations, and swallowing exercises. The positive effects of these strategies have been reported. For swallowing exercises, Robbins et al. (2007) reported that there are temporal characteristics in poststroke survivors after 8-week lingual exercises. Results indicated that the pharyngeal response duration and oral transit duration improved after 8 weeks of lingual exercises in poststroke survivors with dysphagia [23]. Post-stroke survivors with thickened liquids and postural changes such as chin down showed a reduced frequency of aspiration $[24,25]$. The chin down posture helps increase stability during the duration of the laryngeal closure [26]. Thermal stimulation significantly reduced the duration of stage transition (DST) and total swallow duration (TSD) in post-stroke survivors [27]. Reduced duration of stage transition indicates that as the bolus enters into the pharynx, hyolaryngeal excursion, which results in laryngeal closure, quickly occurs. The findings of this study provide additional evidence to support the effectiveness of traditional swallowing intervention on the initiation of laryngeal closure.

This study revealed that the duration of laryngeal closure was slightly prolonged in the follow-up study, although the change of laryngeal closure duration was not significant. In addition, the laryngeal closure duration in this study for both the initial and follow-up VFSS did not reach the range of normal older individuals ( 0.59 seconds) [9]. Three to five weeks of swallowing intervention may not be long enough to show improvement of laryngeal closure duration. It is necessary to follow up the laryngeal closure duration after more than one month of intervention to expect improvement.

Neuroplasticity is the brain's ability to adjust or compensate for lesions to certain areas that result in deficits $[28,29]$. If dysphagia is a result of a stroke lesion to the brain, other areas of the patient's brain may compensate for the affected area to produce a normal swallow. Hussain, Woolfrey, Massey, Geddes, \& $\operatorname{Cox}$ (1996) reported that in post-stroke survivors whose swallowing did not improve two weeks after the onset of their stroke, would improve at a mean of 69 days following the stroke based on neuroplasticity [30]. Taking neuroplasticity into consideration, the laryngeal closure duration may still be in the process of change in these stroke survivors. It is possible that the recovery of laryngeal closure duration takes more than one month. In addition, this study suggests that the initiation of laryngeal closure is recovered earlier than the duration of laryngeal closure. Further research may investigate to integrate the temporal characteristics of airway protection and brain recovery in post-stroke survivors.

This investigation has several limitations including the small sample size, uncontrolled lesion location and size of the stroke, and a short-term follow-up. In addition, we should be careful to interpret the outcomes of this study. Post-stroke survivors in this study had a period of spontaneous recovery. It is unknown whether early intervention will facilitate effective swallowing intervention. It is necessary to have a control group to understand how swallowing recovers in these populations. An investigation on comparative effectiveness of swallowing intervention strategies to improve laryngeal closure is suggested. Future studies with large numbers of patients are warranted, with longitudinal observation using more temporal and biomechanical measurements and clinical observation.

\section{CONCLUSIONS}

This study reported improvement of airway protection after traditional swallowing intervention in post-stroke survivors. The post-stroke survivors initiated the laryngeal closure quicker after swallowing intervention. The findings of this investigation support the effectiveness of the swallowing intervention using temporal measurements of laryngeal closure. In addition, this study suggested the use of temporal measurement of laryngeal closure as a tool for clinicians to evaluate progress during intervention.

\section{REFERENCES}

1. Kidd D, Lawson J, Nesbitt R. Aspiration in acute stroke: a clinical study with videofluoroscopy. QJM: An International Journal of Medicine. 1993;86:825-829.

2. Daniels SK, Brailey K, Priestly DH. Aspiration in patients with acute stroke. Archives of Physical Medicine and Rehabilitation. 1998;79:14-19.

3. Alberts MK, Horner J, Gray L, Brazer SR. Aspiration after stroke: lesion analysis by brain MRI. Dysphagia. 1992;7:170-173.

4. Kendall KA, Leonard RJ. Bolus transit and airway protection coordination in older dysphagic patients. Laryngoscope. 2001;111: 2017-2021.

5. Garon BR, Huang Z, Hommeryer M, Eckmann D, Stern GA, Ormiston C. Abnormal epiglottic movement patterns. Dysphagia. 2002;17:57-68.

6. Perlman AL, Booth BM, Grayhack JP. Videofluoroscopic predictors of aspiration in patients with oropharyngeal dysphagia. Dysphagia. 1994;9:90-95.

7. Han H, Shin G, Jun A, Park T, Ko D, Choi E, et al. The relation be- 
tween the presence of aspiration or penetration and the clinical indicators of dysphagia in poststroke survivors. Annals of Rehabilitation Medicine. 2016;40:88-94.

8. Gordon C, Hewer RL, Wade DT. Dysphagia in acute stroke. Br Med J (Clin Res Ed). 1987;295:411-414.

9. Park T, Kim Y, Ko DH, McCullough GH. Initiation and duration of laryngeal closure during the pharyngeal swallow in post-stroke patients. Dysphagia. 2010;25:177-182.

10. Power ML, Hamdy S, Singh S, Tyrrell PJ, Turnbull I, Thompson DG. Deglutitive laryngeal closure in stroke patients. Journal of Neurology, Neurosurgery, Psychiatry. 2007;78:141-146.

11. Robbins J, Levine RL, Maser A, Rosenbek JC, Kempster GB. Swallowing after unilateral stroke of the cerebral cortex. Archives of physical medicine and rehabilitation. 1993;74:1295-1300.

12. Park T, Kim Y, McCullough G. Oropharyngeal transition of the bolus in post-stroke patients. American Journal of Physical Medicine \& Rehabilitation. 2013;92:320-326.

13. Smithard DG, O'Neil PA, England RE, Park CL, Wyatt R, Martin $\mathrm{DF}$, et al. The natural history of dysphagia following a stroke. Dysphagia. 1997;12:188-193.

14. Barer DH. The natural history and functional consequences of dysphagia after hemispheric stroke. Journal of Neurology, Neurosurgery \& Psychiatry. 1989;52:236-241.

15. Mann G, Hankey GJ, Cameron D. Swallowing function after stroke. Prognosis and prognostic factors at 6 month. Stroke. 1999;30:744748.

16. Terre R, Mearin F. Oropharyngeal dysphagia after the acute phase of stroke: predictors of aspiration. Neurogastroenterology \& Motility. 2006;18:200-205.

17. Terre R, Mearin F. Resolution of tracheal aspiration after acute phase of stroke-related oropharyngeal dysphagia. The American College of Gastroenterology. 2009;104:923-929.

18. Seo HG, Oh BM, Han TR. Longitudinal changes of the swallowing process in subacute stroke patients with aspiration. Dysphagia. 2011;26:41-48.

19. Kendall KA, Leonard RJ, McKenzie S. Airway protection: evaluation with videofluoroscopy. Dysphagia. 2004;19:65-70.
20. Logemann JA, Pauloski BR, Rademaker AW, Kahrilas PJ. Oropharyngeal swallow in younger and older women: videofluoroscopic analysis. Journal of speech, Language, and Hearing Research. 2002;45:434-445.

21. Logemann JA, Pauloski BR, Rademaker AW, Colangelo LA, Kahrilas PJ, Smith $\mathrm{CH}$. Temporal and biomechanical characteristics of oropharyngeal swallow in younger and older men. Journal of Speech, Language, and Hearing Research. 2000;43:1264-1274.

22. Park T, Kim Y, Oh BM. Laryngeal closure during swallowing in stroke survivors with cortical or subcortical lesion. Journal of Stroke and Cerebrovascular Diseases. 2017;26:1766-1772.

23. Robbins J, Kays SA, Gangnon RE, Hind JA, Hewitt AL, Gentry LR, et al. The effects of lingual exercise in stroke patients with dysphagia. Archives of Physical Medicine and Rehabilitation. 2007;88: 150-158.

24. Ney DM, Weiss JM, Kind AJ, Robbins J. Senescent swallowing: impact, strategies, and interventions. Nutrition in Clinical Practice. 2009;24:395-413.

25. National Dysphagia Diet Task Force \& American Dietetic Association. National dysphagia diet: standardization for optimal care. American Dietetic Association. 2002.

26. Macrae P, Anderson C, Humbert I. Mechanisms of airway protection during chin-down swallowing. Journal of Speech, Language, and Hearing Research. 2014;57:1251-1258.

27. Rosenbek JC, Roecker EB, Wood JL, Robbins J. Thermal application reduces the duration of stage transition in dysphagia after stroke. Dysphagia. 1996;11:225-233.

28. Barritt AW, Smithard DG. Role of cerebral cortex plasticity in the recovery of swallowing function following dysphagic stroke. Dysphagia. 2009;24:83-90.

29. Hamdy S, Rothwell J C, Aziz Q, Thompson DG. Organization and reorganization of human swallowing motor cortex: implications for recovery after stroke. Clinical Science. 2000;98:151-157.

30. Hussain A, Woolfrey S, Massey J, Geddes A, Cox J. Percutaneous endoscopic gastrostomy. Postgraduate Medical Journal. 1996;72: 581-585. 